\title{
HAMILTONIAN SYSTEMS: STABILITY AND INSTABILITY THEORY
}

\author{
PATRICK BERNARD
}

The solar system has long appeared to astronomers and mathematicians as a model of stability. On the other hand, statistical mechanics relies on the assumption that large assemblies of particles form highly unstable systems (at the microscopic scale). Yet all these physical situations are described, at least to a certain degree of approximation, by Hamiltonian systems.

One may hope that Hamiltonian systems can be classified in two different categories, stable and unstable ones. However, the situation is much more complicated and both stable and unstable behaviors cohabit in typical systems. Even our examples are not perfect paradigms of stability and instability. Indeed, it is now clear from numerical as well as theoretical points of views that some instability is present over long time-scales in the solar systems, so that for example future collisions between planets can not be completely ruled out in view of our present understanding. On the other hand, unexpected patterns of stability have been discovered in systems involving a large number of particles.

Understanding the impact of stable and unstable effects in Hamiltonian systems has been considered since Poincaré as one of the most important questions in dynamical systems. In the present text, we will discuss model Hamiltonian systems of the form

$$
H_{\epsilon}(q, p)=h(p)+\epsilon G_{\epsilon}(q, p)
$$

where $(q, p) \in \mathbb{T}^{d} \times U$, with $U$ a bounded open subset of $\mathbb{R}^{n}$. Recall that the equations of motion are

$$
\begin{gathered}
\dot{q}(t)=\partial_{p} h(p)+\epsilon \partial_{p} G_{\epsilon}(q, p) \\
\dot{p}(t)=-\epsilon \partial_{q} G_{\epsilon}(q, p) .
\end{gathered}
$$

The textbook [1] is a good general introduction on Hamiltonian systems. We will always denote by $\omega(p)$ the frequency map $\partial_{p} h(p)$, which plays a crucial role. Here, as is obvious in (2), the action variables $p$ are preserved under the evolution in the unperturbed case $\epsilon=0$. We will try to explain what is known on the evolution of these action variables for the perturbed system. As we will see, in many situations, these variables are extremely stable. For example, KAM theorem implies that, for a positive measure of initial conditions $\left(q_{0}, p_{0}\right)$ the trajectory $(q(t), p(t))$ satisfies $\|p(t)-p(0)\| \leqslant C \epsilon$ for all times. Examples show that some initial conditions may lead to unstable trajectories, that is trajectories such that $\|p(t)-p(0)\| \geqslant 1 / C$ for some $t$ (depending on $\epsilon$ ) and some fixed constant $C$ independent of $\epsilon$. However, this is, as we will see, possible only for very large time $t$ (meaning that $t$ as a function of $\epsilon$ has to go to infinity very quickly when $\epsilon \longrightarrow 0$ ). The main questions here are to understand in what situation instability is or is not possible, and what kind of evolutions can have the actions variable $p$. Another important question is to estimate the speed (as a function of the parameter $\epsilon$ ) of the evolutions of $p$. 
0.1. A convention. We assume, unless otherwise stated, that the Hamiltonians are real analytic. The norm $|H|$ of the Hamiltonian $H$ is the uniform norm of its holomorphic extension to a certain complex strip. We do not specify the width of this strip. Whenever we consider a family $H_{\epsilon}, F_{\epsilon} \ldots$ of Hamiltonians, we mean that the norm $\left|H_{\epsilon}\right|$ is bounded when $\epsilon \longrightarrow 0$.

\section{Averaging AND EXPONENTIAL StABILITY.}

The first observation concerning the action variable is that they should evolve at a speed of the order of $\epsilon$. However, averaging effects occur. More precisely, in the equation $\dot{p}(t)=$ $-\epsilon \partial_{q} H_{\epsilon}(q(t), p(t))$, the variable $q(t)$ is moving fast compared to $p(t)$. If the evolution of $q(t)$ nicely fills the torus $\mathbb{T}^{n}$, it is tempting to think that the averaged equation

$$
\dot{\bar{p}}(t)=-\epsilon \bar{V}_{\epsilon}(\bar{p}(t))
$$

should approximate accurately the actual behavior of $p(t)$, where

$$
\bar{V}_{\epsilon}(p):=\int_{\mathbb{T}^{d}} \partial_{q} H(q, p) d q .
$$

We have $\bar{V} \equiv 0$, which leads to think that the evolution should consist mainly of oscillations of small amplitude with no large evolution. This reasoning is limited by the presence of resonances.

1.1. Frequencies. A frequency $\omega \in \mathbb{R}^{d}$ is said resonant if there exists $k \in \mathbb{Z}_{*}^{d}\left(=\mathbb{Z}^{d}-\{0\}\right)$ such that $\langle k, \omega\rangle=0$. The resonance module of $\omega$,

$$
Z(\omega)=\left\{k \in \mathbb{Z}^{d} /\langle k, \omega\rangle=0\right\}
$$

is a subgroup $\mathbb{Z}^{d}$, we denote by $R(\omega)$ the vector space generated by $Z(\omega)$ in $\mathbb{R}^{d}$. The order of resonance $r(\omega)$ is the dimension of $R(\omega)$. The main examples of a resonances of order $r$ are the frequencies $\omega=\left(\omega_{1}, 0\right)$ where $\omega_{1} \in \mathbb{R}^{d-r}$ is non resonant. This example is universal. Indeed, if $\omega$ is a resonant frequency, then there exists a matrix $A \in G l_{d}(\mathbb{Z})$ such that $A \omega=\left(\omega_{1}, 0\right)$, where $\omega_{1} \in \mathbb{R}^{d-r}$ is not resonant. The matrix $A$ can be seen as a diffeomorphism of $\mathbb{T}^{d}$, which transports the constant vector-field $\omega$ to the constant vector-field $A \omega=\left(\omega_{1}, 0\right)$. It is useful to distinguish, among non resonant frequencies, some which are sufficiently non-resonant. A frequency $\omega \in \mathbb{R}^{d}$ is called Diophantine if there exists real constants $\gamma>0$ and $\tau \geqslant d$ such that

$$
|\langle\omega, k\rangle| \geqslant \gamma\|k\|^{1-\tau}
$$

for each $k \in \mathbb{Z}_{*}^{d}$. Finally, a frequency is called resonant-Diophantine if there exists a matrix $A \in G l_{n}(\mathbb{Z})$ such that $\omega=A\left(\omega_{1}, 0\right)$, where $\omega_{1} \in \mathbb{R}^{d_{1}}$ is a Diophantine frequency.

1.2. Symplectic diffeomorphisms and Normal Forms. An efficient mathematical method to take averaging effects into account is the use of normal forms. Normal form theory consists in finding new coordinates in which the fast angles have been eliminated from the equations up to a small remainder. This is done exploiting the existence of a large group of diffeomorphisms preserving the Hamiltonian structure of equations, called symplectic diffeomorphisms or canonical transformations. We refer the reader to standard textbooks for these notions, for example to [1]. An important point is that a symplectic diffeomorphism $\phi$ sends the trajectories of the Hamiltonian $H \circ \phi$ to the trajectories of the Hamiltonian $H$. A Hamiltonian $N(q, p)$ is said in $R$-normal form, where $R$ is a linear subspace of $\mathbb{R}^{n}$, if $\partial_{q} N \in R$ for each $(q, p)$. Let us give an illustrative result, taken from [8]. Note that this result is not sufficient to 
obtain uniform stability estimates, as in Nekhoroshev Theorem below. More precise normal form results are given in [9] and [11].

1.3. Normal form theorem. Let $\omega_{0}=\omega\left(p_{0}\right)$ be a given Diophantine or resonant-Diophantine frequency. Let us denote $B_{r}\left(p_{0}\right)$ the open ball of radius $r$ in $\mathbb{R}^{d}$ centered at $p_{0}$. There exists a constant a which depends only on $\omega$, and constants $\epsilon_{0}>0$ and $C>0$ such that the following holds: For each $\epsilon<\epsilon_{0}$, there exists an analytic symplectic embedding $\phi_{\epsilon}: \mathbb{T}^{d} \times B_{r(\epsilon)} \longrightarrow \mathbb{T}^{d} \times U$, which is $\epsilon$-close to identity and such that

$$
H_{\epsilon} \circ \phi_{\epsilon}(q, p)=h(p)+\epsilon N_{\epsilon}(q, p)+\mu(\epsilon) F_{\epsilon}(q, p),
$$

where $N$ is in $R\left(\omega_{0}\right)$-normal form, $r(\epsilon) \geqslant \sqrt{\epsilon}$, and $\mu(\epsilon) \leqslant e^{-C \epsilon^{-a}}$.

This means that the motions with resonant initial conditions are confined, up to small oscillations, in the associated affine plane $p(0)+R(\omega(p(0))$ until they live the domain of the normal form, or until time $\mu^{-1}(\epsilon)$.

1.4. Geometry of resonances. In view of the Normal Form Theorem, we are led to consider the curves $P(\theta): \mathbb{R} \longrightarrow \mathbb{R}^{d}$ which satisfy

$$
P\left(\theta^{\prime}\right)-P(\theta) \in R(\omega(P(\theta)))
$$

for each $\theta$ and $\theta^{\prime}$. Indeed, it appears that these curves are the ones the action variables can follow on time-scales not involving the remainders of the normal forms. Note that here the parameter $\theta$ is not the physical time. Assuming that $P(\theta)$ is such a curve, we can define the affine space

$$
R:=P(0)+\cap_{\theta \in \mathbb{R}} R(\omega(P(\theta))) .
$$

We then have $P(\theta) \in R$ for each $\theta$. In addition, each point $P(\theta), \theta \in \mathbb{R}$ is a critical point of the restriction $h_{\mid R}$ of the unperturbed Hamiltonian $h$ to the affine space $R$. It follows that the curve $P(\theta)$ has to be constant if the unperturbed Hamiltonian satisfies the following hypothesis.

1.5. Nekhoroshev steepness. We say that the unperturbed Hamiltonian $h$ is steep if, for each affine subspace $\Lambda$ in $\mathbb{R}^{d}$, the restriction $h_{\mid \Lambda}$ has only isolated critical points.

This formulation, due to L. Niederman, [10], is much simpler than the equivalent one first given by Nekhoroshev in [9]. It turns out that this condition, which was made natural by our heuristic explanation, implies stability over exponential time-scales for all initial conditions, see [9]. We first need another condition.

1.6. Kolmogorov non-degeneracy. We say that the unperturbed Hamiltonian $h$ is nondegenerate in the sense of Kolmogorov if it has non-degenerate Hessian at each point, or equivalently if the frequency map $p \longmapsto \omega(p)$ is an immersion.

1.7. Nekhoroshev stability theorem. Assume that the unperturbed Hamiltonian does not have critical points $(\omega(p)$ does not vanish), satisfies Nekhoroshev steepness and Kolmogorov non-degeneracy conditions. Then there exists constants $a>0$ and $b>0$, which depend only on $h$, and constants $\epsilon_{0}>0$ and $C>0$ such that the following holds: For $\epsilon<\epsilon_{0}$, each trajectory $\left(q_{\epsilon}(t), p_{\epsilon}(t)\right)$ satisfies the estimate

$$
\left\|p_{\epsilon}(t)-p_{\epsilon}(0)\right\| \leqslant C \epsilon^{b}
$$

for all $t$ such that $|t| \leqslant e^{C \epsilon^{-a}}$. 
1.8. Herman's example. In oder to illustrate the necessity of the condition of steepness, let us consider the Hamiltonian

$$
H_{\epsilon}\left(q_{1}, q_{2}, p_{1}, p_{2}\right)=p_{1} p_{2}+\epsilon V\left(q_{1}\right) .
$$

with $V: \mathbb{T} \longrightarrow \mathbb{R}$. The associated equations are

$$
\dot{p}_{2}=0, \quad \dot{p}_{1}=-V^{\prime}, \quad \dot{q}_{1}=p_{2}, \quad \dot{q}_{2}=p_{1} .
$$

The trajectories whose initial condition are subjected to $p_{2}(0)=0$ and $V^{\prime}\left(q_{1}(0)\right) \neq 0$ satisfy

$$
p_{1}(t)=p_{1}(0)-t \epsilon V^{\prime}\left(q_{1}(0)\right), \quad p_{2}(t)=0, \quad q_{1}(t)=q_{1}(0) .
$$

We see an evolution at speed $\epsilon$ of the action variable $p_{1}$ contradicting the conclusion of Nekhoroshev theorem. In this example, we have $R(\omega(p(t)))=\mathbb{R} \times\{0\}$, and $h_{\mid \mathbb{R} \times\{0\}} \equiv 0$, so that the curve

$$
P(\theta)=(\theta, 0)
$$

is indeed a curve of critical points of $h_{\mid \mathbb{R} \times\{0\}}$.

1.9. Genericity of steepness. The condition of steepness is frequently satisfied. In order to be more precise, we mention that, for $N \in \mathbb{N}$ large enough (how large depends on the dimension $d$ ), steepness is a generic condition in the finite dimensional space of polynomials of degree less that $N$. Note in contrast that a quadratic Hamiltonian is steep if and only if it is positive definite. Finally, it is important to mention that convex Hamiltonians $h$ with positive definite Hessian are steep. More generally, quasi-convex Hamiltonians are steep. A function $h: U \longrightarrow \mathbb{R}$ is said quasi-convex if, at each point, the restriction of its Hessian to the kernel of its differential is positive definite.

1.10. The quasi-convex case. It is interesting to be more precise about the values of $a$ and $b$ in Nekhoroshev Theorem. We shall do so in the quasi-convex case, which is the most stable case, and where much more is known. If $h$ is quasi-convex, one can take

$$
a=b=\frac{1}{2 d},
$$

as was proved by P. Lochak, see [7]. It is a question of active present research whether these exponents are optimal. It now appears that this is almost so, and that the optimal exponent $a$ should not be larger than $1 / 2(d-3)$. That this exponent deteriorates as the dimension increases is of course very natural in the perspective of statistical mechanics. As a matter of fact, not only the exponent $a$, but also the threshold $\epsilon_{0}$ of validity of Nekhoroshev Theorem deteriorates with the dimension, as was noticed in [5].

Another important fact was proved in [7]: in these expressions, the important value of $d$ is not the total number of degrees of freedom, but the number of active degrees of freedom. More precisely, resonant initial condition are more stable than generic ones. If $r$ is the order of resonance of a given initial condition, then the number $d-r$ of fast angles can be substituted to the total number of degrees of freedom for the computation of the stability exponent. This phenomenon may account for the surprising stability obtained numerically by Fermi, Pasta and Ulam.

\section{Permanent stability.}

Many initial conditions satisfy more than exponential stability: they are permanently stable. 
2.1. Kolmogorov Theorem. Assume that h satisfies Kolmogorov non-degeneracy condition 1.6. Then for each open subset $V \subset \mathbb{R}^{d}$ such that $\bar{V} \subset U$, there exists $\epsilon_{0}>0$ such that, for each $\epsilon<\epsilon_{0}$ there exists

- a smooth symplectic embedding $\phi_{\epsilon}: \mathbb{T}^{d} \times V \longrightarrow \mathbb{T}^{d} \times U$, which is $\epsilon$-close to the identity, - a compact subset $F_{\epsilon}$ of $V$, whose relative measure in $V$ is converging to 1 as $\epsilon \longrightarrow 0$, such that the Hamiltonian system $H_{\epsilon} \circ \phi_{\epsilon}$ preserves the torus $\mathbb{T}^{d} \times\{p\}$ for each $p \in F_{\epsilon}$.

The union

$$
\mathcal{F}_{\epsilon}=\phi_{\epsilon}\left(\mathbb{T}^{d} \times F_{\epsilon}\right)
$$

of all the invariant tori has positive measure. Its complement is usually an open dense subset of $\mathbb{T}^{d} \times U$. All the orbits starting in this invariant set obviously undergo oscillations of amplitude of the order of $\epsilon$ for all times. It is worth mentioning that some energy surfaces may not intersect the invariant set $\mathcal{F}_{\epsilon}$. This is illustrated in example 1.8, where the surface of zero energy does not contain invariant tori. The following condition guaranties the existence of invariant tori on each energy surface.

2.2. Arnold non-degeneracy. The Hamiltonian $h$ is said to be non-degenerate in the sense of Arnold if it does not have critical points and if the map

$$
p \longmapsto \frac{\omega(p)}{\|\omega(p)\|}
$$

is a local diffeomorphism between each level set of $h$ and $S^{d-1}$. This is equivalent to say that the function $(\lambda, p) \in \mathbb{R} \times U \longmapsto \lambda h(p)$ has non-degenerate Hessian at each point of the form $(1, p)$.

2.3. Arnold Theorem. If $h$ satisfies Arnold non-degeneracy condition, then the relative measure of the set $\mathcal{F}_{\epsilon}$ of invariant tori is converging to 1 in each energy surface.

This theorem prevents ergodicity of the perturbed systems for the canonical invariant measure on its energy surface. This may be considered as a very disappointing result for statistical mechanics, whose mathematical foundation has often be considered to be the Boltzmann hypothesis of ergodicity. However, statistical mechanics is first of all a question of letting $d$ go to infinity, and ergodicity might not be such a crucial hypothesis, see [6].

When $d=2$, The theorem of Arnold has particularly strong consequences. Indeed, in this case, the invariant tori cut the energy surfaces in small connected components. The motion is then confined in these connected components. As a consequence, we obtain permanent stability for all initial conditions.

In higher dimension however, the complement of $\mathcal{F}_{\epsilon}$ in each energy shell is usually a dense, connected open set. There may exist orbits wandering in this large connected set, although the speed of evolution of these orbits is limited by Nekhoroshev theory. Understanding the dynamics in this open set is a very important and hard question. It is the subject of the next section.

2.4. Relaxed assumption. For many applications, such as celestial mechanics, the nondegeneracy conditions of Arnold or Kolmogorov are not satisfied, or hard to check. However, the existence of invariant tori has been proved under much milder assumptions. As a rule, Invariant tori exist in the perturbed systems if the frequency map $p \longmapsto \omega(p)$ stably contains Diophantine vectors in its image. 


\section{The Mechanism of Arnold}

Understanding instability is the subject of intense present research. General methods of construction of interesting orbits, as well as clever classes of examples are being developed. These methods are exploring the limits of stability theory. Here we shall only describe the foundational ideas of Arnold, see [1], where most of the present activity finds its roots. Although these ideas have some ambition of universality, they are best presented, like in [1], on an example. We consider the quasi-convex Hamiltonian

$$
H\left(q_{1}, q_{2}, q_{3}, p_{1}, p_{2}, p_{3}\right)=\left(p_{1}^{2}+p_{2}^{2}\right) / 2-p_{3}+\epsilon \cos 2 \pi q_{2}+\mu\left(\cos 2 \pi q_{2}\right)\left(\cos 2 \pi q_{1}+\cos 2 \pi q_{3}\right) .
$$

As we have seen, this system is typical of the kind of Hamiltonians one gets after reduction to resonant normal form. However, it is illuminating to consider $\mu$ not as a function of $\epsilon$ but as an independent parameter. This is an idea of Poincaré then followed by Arnold. We shall expose the main steps of the proof of the following result.

3.1. Theorem. Let us fix numbers $0<A<B$. For each $\epsilon>0$, there exists a number $\mu_{0}(\epsilon)$ such that, when $0<\mu<\mu_{0}(\epsilon)$, there exists a trajectory

$$
\left(q_{1}(t), q_{2}(t), p_{1}(t), p_{2}(t)\right)
$$

and a time $T>0$ (which depends on $\epsilon$ and $\mu$ ) such that

$$
p_{1}(0) \leqslant A, p_{1}(T) \geqslant B \text {. }
$$

3.2. The truncated system. Let us begin with some remarks about the truncated Hamiltonian obtained when $\mu=0$ :

$$
H_{0}(q, p)=H_{1}\left(q_{1}, q_{3}, p_{1}, p_{3}\right)+H_{2}\left(q_{2}, p_{2}\right)=p_{1}^{2} / 2-p_{3}+p_{2}^{2} / 2+\epsilon \cos 2 \pi q_{2} .
$$

This system is the uncoupled product of $H_{1}$ and of the pendulum described by $H_{2}$. The variable $p_{1}$ is constant along motion, hence the Theorem can not hold for $\mu=0$.

Recall that the point $q_{2}=0, p_{2}=0$ is a hyperbolic fixed point of the pendulum $H_{2}\left(q_{2}, p_{2}\right)=$ $p_{2}^{2} / 2+\epsilon \cos 2 \pi q_{2}$. The stable and unstable manifolds of this integrable system coincide, they from the energy level $H_{2}=\epsilon$. As a consequence, in the product system of Hamiltonian $H_{0}=H_{1}+H_{2}$, there exists, in the zero energy level, a one parameter family $T_{\omega}$ of invariant tori of dimension 2

$$
T_{\omega}=\left\{p_{1}=\omega, p_{3}=\omega^{2} / 2+\epsilon, q_{2}=0, p_{2}=0\right\} \subset \mathbb{T}^{3} \times \mathbb{R}^{3} .
$$

Each of these tori is hyperbolic in the sense that it has a stable manifold of dimension 3 and an unstable manifold of dimension 3 , which are nothing but the liftings of the stable and unstable manifolds of the hyperbolic fixed point of $H_{2}$. Notice that these manifolds do not intersect transversally along $T_{\omega}$.

When $\mu \neq 0$, the perturbation is chosen in such a way that the tori $T_{\omega}$ are left invariant by the Hamiltonian flow. 
3.3. Splitting. For $0<\mu<\mu_{0}(\epsilon)$, the invariant tori $T_{\omega}$ still have stable and unstable manifolds of dimension 3. These stable and unstable manifolds intersect transversally in the energy surface, along an orbit which is homoclinic to the torus.

The first point is that the tori remain hyperbolic, and that the stable and unstable manifolds are deformed, but not destroyed by the additional term. This results from the observation that the manifold $M$ formed by the union of the invariant tori is normally hyperbolic in its energy surface. Note that this step does not require exponential smallness of $\mu$.

It is then a very general result that the stable and unstable manifolds have non-empty intersection. It is a global property, which can be established by variational methods, and which still does not rest on exponential smallness of $\mu$.

The key point, where exponential smallness is required, is transversality. Since transversality is a generic phenomenon, one may think that this step is not so crucial. And indeed, it is very likely that the statement remains true for most values of $\mu \in] 0, \epsilon$ ] (and not only for $\left.\mu \leqslant \mu_{0}(\epsilon)\right)$. However, there are two important issues here. First, transversality is hard to establish on explicit examples. Second, it is useful for many further discussions to obtain some quantitative estimates.

Indeed, we can associate to the intersection between the stable and unstable manifolds a quantity, the splitting, which in a sense measures transversality. Discussions on such a definition are available in [8]. Using methods of Poincaré and Melnikov, Arnold showed that this splitting can be estimated, for sufficiently small $\epsilon$, by

$$
\alpha \geqslant \mu e^{-C / \sqrt{\epsilon}}+O\left(\mu^{2}\right) \text {. }
$$

This implies non-nullity of the splitting, hence transversality, for small $\mu$.

3.4. Transition chain. We have established the existence, when $\mu>0$ is small enough, of a family $T_{\omega}$ of hyperbolic invariant tori such that the stable manifold $W_{\omega}^{+}$and the unstable manifold $W_{\omega}^{-}$intersect transversally along a homoclinic orbit (but not along $T_{\omega}$ !) for each $\omega$.

A stability argument shows that the stable manifold $W_{\omega}^{+}$of the torus $T_{\omega}$ intersects transversally the stable manifold $W_{\omega_{0}}^{-}$of the torus $T_{\omega_{0}}$ when $\omega$ is close enough to $\omega_{0}$. How close directly depends on the size of the splitting. We obtain heteroclinic orbits between tori close to each other.

Given two values $\omega$ and $\omega^{\prime}$, we can find a sequence $\omega_{i}, 1 \leqslant i \leqslant N$ such that $\omega_{0}=\omega$, $\omega_{N}=\omega^{\prime}$, and $W_{i}^{-}$intersects transversally $W_{i+1}^{+}$for all $i$. The associated family $T_{\omega_{i}}$ of tori is called a transition chain.

The left step consists in proving that some orbits shadow the transition chain. Arnold solved this step by a very simple topological argument which, however, does not provide any estimate on the time $T$. He proves the existence of an orbit joining any neighborhood of $T_{\omega}$ to any neighborhood of $T_{\omega^{\prime}}$. This ends the proof of the main theorem, since we can chose $\omega$ and $\omega^{\prime}$ such that $\omega<A<B<\omega^{\prime}$.

The dynamics associated to hyperbolic tori and transition chains have later been studied more carefully. It particular, a $\lambda$-lemma can be proved in this context, which allows to conclude that, in a transition chain, the unstable manifold $W_{0}^{-}$of the first torus intersects transversally the stable manifold of the last torus $W_{N}^{+}$. These detailed study also allow to relate the speed of diffusion to the splitting of the invariant manifolds.

3.5. Diffusion speed. It is interesting to estimate the speed of evolution of the variable $p_{1}$, or in other words the time $T$ in the statement. It follows from Nekhoroshev theory that this time $T$ has to be exponentially large as a function of $\epsilon$. In fact, it is possible to prove, either 
by recent developments on the ideas of Arnold exposed above, or more easily by variational methods, 4], that

$$
T \leqslant \frac{e^{C / \sqrt{\epsilon}}}{-\mu \log \mu}
$$

for $\mu \leqslant \mu_{0}(\epsilon)$. This time is of course highly related to the estimate (3) of the splitting. In addition, Ugo Bessi proved that one can take $\mu_{0}(\epsilon)=e^{-C / \sqrt{\epsilon}}$. Plugging this value of $\mu$ in the estimate of $T$, we get the estimate $T \leqslant e^{C / \sqrt{\epsilon}}$ as a function of the only parameter $\epsilon$. Considering the fact that the orbit we have described go close to double resonances, this is the best estimate one may hope for in view of the improved Nekhoroshev stability estimates at resonances.

The idea is now well spread that the time of diffusion is exponentially large. However, we point out that, if it is indeed exponentially small as a function of the parameter $\epsilon$, it is only polynomially small as a function of the second parameter $\mu$, as was first understood by $\mathrm{P}$. Lochak and proved in [3] using the variational method of U. Bessi.

3.6. Conclusion. The theories of instability are developing in several directions. One of them is to try to understand the limits of stability, and to test to what extent the stability results obtained so far are optimal. This aspect has quickly developed recently, for example the optimal stability exponent $a$ for convex systems is almost known. Another direction is to try to give a description of unstable orbits in typical systems. This remains a widely open question.

Let us finally mention that the application of the theories we have presented to concrete systems is very difficult. One of the reasons is that the estimates of the threshold $\epsilon_{0}$ of validity of Nekhoroshev and KAM theorems that can (painfully) be obtained by inspection in the proofs are very bad. Much too bad, for example, to think about applications to the solar systems with the physical values of the parameters.

\section{REFERENCES}

[1] V. I. Arnold, Instability of dynamical systems with several degrees of freedom. Sov. Math. Doklady, 5 (1964), 581-585.

[2] V. I. Arnold, Mathematical methods of classical mechanics, Springer.

[3] P. Bernard, Perturbation d'un hamiltonien partiellement hyperbolique, Comptes Rendus de l'Academie des sciences, serie I, 323 (1996).

[4] U. Bessi, An approach to Arnold's diffusion through the calculus of variations. Nonlinear Anal. 26, no. 6 (1996), 1115-1135.

[5] J. Bourgain and V. Kaloshin On diffusion in high-dimensional Hamiltonian systems, Journal of Functional Analysis, in press.

[6] A. I. Khinchin Mathematical Foundations of Statistical Mechanics, Dover Publications.

[7] P. Lochak, Canonical perturbation theory via simultaneous approximation, Russian Math. Surveys 47 (1992), 57-133.

[8] P. Lochak, J. P. Marco, D. Sauzin On the splitting of invariant manifolds in multidimensional near-integrable Hamiltonian systems. Mem. Amer. Math. Soc. 163 (2003), no. 775,

[9] N.N.Nekhoroshev, An exponential estimate for the time of stability of nearly integrable Hamiltonian systems, Russian Math. Surveys 32 (1977), 1-65.

[10] L. Niedreman, Hamiltonian stability and subanalytic geometry, Ann. Inst. Fourier, in press.

[11] J. Pöschel, Nekhoroshev estimates for quasi-convex Hamiltonian systems, Math. Z. 213 (1993), 187216. 